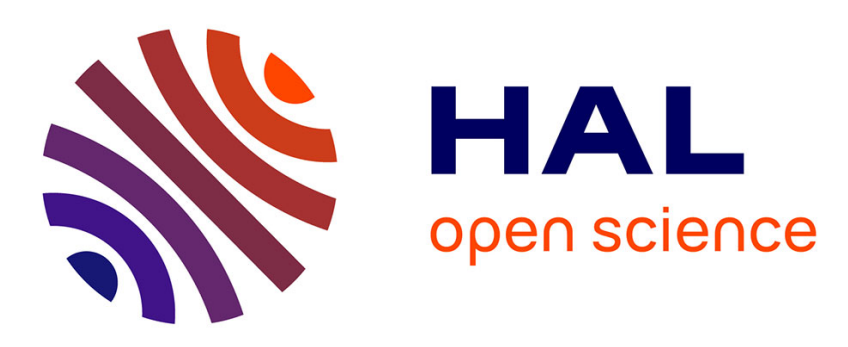

\title{
Artificial intelligence control applied to drag reduction of the fluidic pinball
}

Guy y Cornejo Maceda, Bernd R Noack, François Lusseyran, Nan Deng, Luc Pastur, Marek Morzynski

\section{- To cite this version:}

Guy y Cornejo Maceda, Bernd R Noack, François Lusseyran, Nan Deng, Luc Pastur, et al.. Artificial intelligence control applied to drag reduction of the fluidic pinball. PAMM, 2019, 90th Annual Meeting of the International Association of Applied Mathematics and Mechanics (GAMM), 19 (1), pp.e201900268. 10.1002/pamm.201900268 . hal-02398649

\section{HAL Id: hal-02398649 \\ https://hal.science/hal-02398649}

Submitted on 7 Dec 2019

HAL is a multi-disciplinary open access archive for the deposit and dissemination of scientific research documents, whether they are published or not. The documents may come from teaching and research institutions in France or abroad, or from public or private research centers.
L'archive ouverte pluridisciplinaire HAL, est destinée au dépôt et à la diffusion de documents scientifiques de niveau recherche, publiés ou non, émanant des établissements d'enseignement et de recherche français ou étrangers, des laboratoires publics ou privés. 


\title{
Artificial intelligence control applied to drag reduction of the fluidic pinball
}

\author{
Guy Y. Cornejo Maceda ${ }^{1 *}$, Bernd R. Noack ${ }^{2,3,4}$, François Lusseyran ${ }^{2}$, Nan Deng $^{5}$, Luc Pastur $^{5}$, \\ and Marek Morzynski ${ }^{6}$ \\ ${ }^{1}$ LIMSI, CNRS, Univ. Paris-Sud, Université Paris-Saclay, F-91405 Orsay \\ ${ }^{2}$ LIMSI, CNRS, Université Paris-Saclay, F-91405 Orsay \\ ${ }^{3}$ Harbin Institute of Technology, China \\ ${ }^{4}$ Technische Universität Berlin, Germany \\ ${ }^{5}$ IMSIA - UMR9219, ENSTA ParisTech, France \\ ${ }^{6}$ Poznan University of Technology, Poland
}

\begin{abstract}
The aim of our work is to advance a self-learning, model-free control method to tame complex nonlinear flows-building on the pioneering work of Dracopoulous [1]. The cornerstone is the formulation of the control problem as a function optimization problem. The control law is derived by solving a nonsmooth optimization problem thanks to an artificial intelligence technique, genetic programming (GP). Metaparameters optimization of the algorithm and complexity penalization have been our main contribution and have been tested on a cluster of three equidistant cylinders immersed in a incoming flow, the fluidic pinball. The means of control is the independent rotation of the cylinders. GP derived a control law associated to each cylinder in order to minimize the net drag power and managed to outperform past open-loop studies with a $46.0 \%$ net drag power reduction by combining two strategies from literature. This success of MIMO control including sensor history is promising for exploring even more complex dynamics.
\end{abstract}

Copyright line will be provided by the publisher

\section{Introduction}

Feedback turbulence control is a key enabler for flow control in engineering applications. Aerodynamic drag, lift increase, noise reduction and mixing enhancement, to cite a few examples, are essential problems at the heart of economic and environmental issues. However fundamental challenges including high-dimensionality, strong nonlinearities, time-delays from actuation to sensing and frequency crosstalks need to be addressed by feedback turbulence control [2].

The fluidic pinball - three cylinders placed at the vertices of an equilateral triangle, see figure $1-$ is proposed as a control benchmark to face these challenges. Thanks to the independent rotation of the three cylinders and sensors downstream, up to six wake stabilization strategies inspired from literature can be reproduced : phasor control, boat-tailing, base-bleeding, magnus effect and especially high-frequency forcing, low-

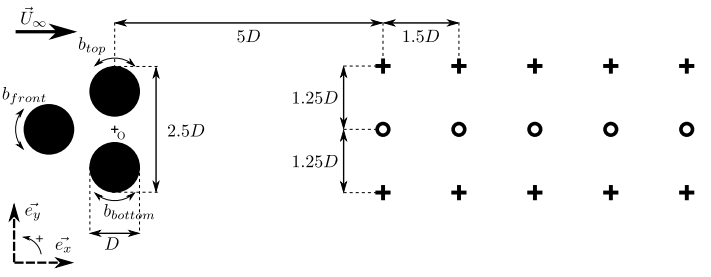

Fig. 1: Fluidic pinball configuration. $b_{\text {front }}, b_{\text {bottom }}$ and $b_{t o p}$ denote the peripheral speed of the cylinders. Circles downstream stand for y-velocity sensors and crosses $\mathrm{x}$-velocity sensors. Axis origin is halfway between the two back cylinders.

frequency forcing attesting for frequency crosstalk. Taming the fluidic pinball shall take into account the richness of these actuation mechanisms.

\section{Artificial intelligence control methodology}

In a general framework, a control law $\vec{K}$ is a function of some feedback quantity $\vec{s}(t)$ and a time-dependent function $\vec{h}(t)$ : $\vec{b}=\vec{K}(\vec{s}(t), \vec{h}(t))$. The design of the control law $\vec{K}$ can be performed thanks to several control methods including modelbased (ERA/OKID), open-loop strategies (multi-frequency forcing), and closed-loop strategies (direct sensor feeback, adaptive control), including history such as ARMAX. Model-based control victories are in opposition and phasor control and examples including MIMO control and frequency crosstalk are dim.

Thus without any prior knowledge of the system, the control problem is formulated as a regression problem. The control law $\vec{K}$ becomes a function to be optimized following a given control objective. This problem is described by equation (1):

$$
\vec{K}_{\text {opt }}=\operatorname{argmin}_{\vec{K}} J(\vec{K})
$$

$\vec{K}_{\text {opt }}$ being the best mapping between the system outputs $\vec{s}$ and the system inputs $\vec{b}$, that minimize the cost function $J$.

* Corresponding author: e-mail cornejo@ limsi.fr, phone +33687987462 
The control law to be derived is thereby a function living in an infinite dimension space. Solving equation (1) implies the resolution of a non-convex optimization problem with presumably multiple local minimas and discontinuities in the search space. To overcome such a problem, a powerful artificial intelligence technique is deployed : genetic programming (GP). It serves to find near optimum solution by identifying both the parameters and the structure of the control law, allowing not only all the previously mentionned mechanisms and methods but also linear and nonlinear combination of them. The control paradigm is illustrated in figure 2 and is further described in Duriez et al. 2016 [3].

Our contribution has been to optimize the metaparameters of the algorithm to improve the speed of the convergence and the quality of the solution. Also, better performance has been achieved thanks to control law complexity penalization.

\section{The fluidic pinball tamed by AIC}

The optimized genetic programming code has been applied to a 2D fluidic pinball. The simulation has been carried out thanks to the UNS3 solver, a highly optimized code that balances speed and accuracy [4]. Increasingly rich control law spaces have been explored to derive the control law. They include multi-frequency forcing; direct sensor feedback thanks to velocity probes (see figure 1); and sensor history. The most performing control law $\left(b_{\text {front }}=-s(t), b_{\text {bottom }}=1.76-s(t) s(t-1), b_{\text {top }}=\right.$ -1.80 ) is found to be a combination of boat-tailing on the back cylinders and phasor control for the front cylinder. It's worth mentioning that GP acts like a sensor optimizer, selecting only one of the fifteen sensors. This control law achieves a $46.0 \%$ net drag power reduction outperforming previous optimized open-loop control strategies such as boat-tailing (42.7\% net drag power reduction) and leads to a smoother wake, see figure 3.
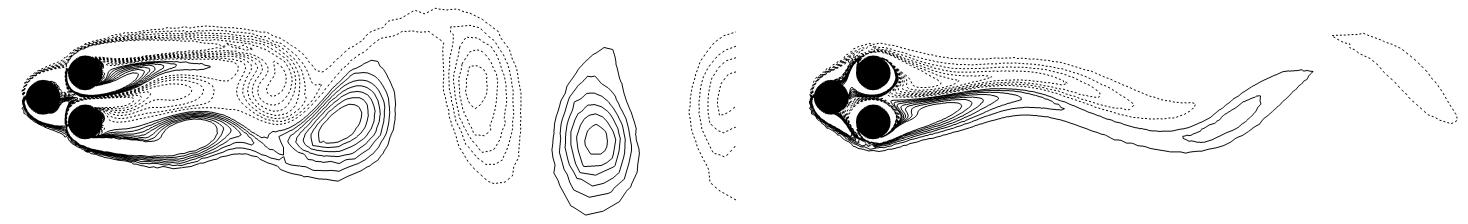

Fig. 3: Contour levels of vorticity for the unforced flow (left) and controlled flow (right) by the control law in section 3. Solid lines and dashed lines represent respectively positive and negative vorticity.

\section{Conclusion and prospectives}

The model-free formulation of the control problem as a non-convex optimization problem enables the exploration of complex, nonlinear dynamics. Such a problem is solved thanks to an iterative stochastic method, genetic programming. An optimization of the metaparameters of the algorithm and complexity penalization of the control law achieved a $46.0 \%$ net drag power reduction on the fluidic pinball combining two strategies from literature: boat-tailing and phasor control. The introduction of sensor history in the search space basis has been the key element to deal with a convective flow as the fluidic pinball. Moreover a large variety of dynamics can be explored depending on the cost function formulation: total fluctuation energy reduction, increase of recirculation bubble length, difference to steady solution are as many different objective to be tested; possibly for one of them leading to a full stabilization of the wake. As for the fluidic pinball, the artificial intelligence control approach has the potential to reveal hidden control mechanisms in real-world experiments, unreachable with model-based approaches, and exploiting nonlinearities and frequency crosstalks.

Acknowledgements Our research is supported by the ASTRID-ANR-17- FLOwCON project, Contrôle d'écoulements turbulents en boucle fermée par apprentissage automatique.

\section{References}

[1] D. Dracopoulos, Evolutionary Learning Algorithms for Neural Adaptive Control, Springer 1997

[2] S. Brunton, B. R. Noack, Closed-Loop Turbulence Control: Progress and Challenges, 2015 Appl. Mech. Rev. 67, 050801

[3] T. Duriez, S. Brunton, B. R. Noack, Machine Learning Control : Taming Nonlinear Dynamics and Turbulence, Springer 2016

[4] B. R. Noack, M. Morzyński, The Fluidic Pinball: A Toolkit for Multiple-Input Multiple-Output Flow Control. Tech. Rep. 02/2017 